\title{
Erratum
}

\section{Erratum: Chen et al., "In Vivo Submillisecond Two-Photon Optogenetics with Temporally Focused Patterned Light"}

In the article "In Vivo Submillisecond Two-Photon Optogenetics with Temporally Focused Patterned Light", by I-Wen Chen, Emiliano Ronzitti, Brian R. Lee, Tanya L. Daigle, Deniz Dalkara, Hongkui Zeng, Valentina Emiliani, and Eirini Papagiakoumou, which appeared on pages 3484-3497 of the May 1, 2019 issue, the funding information in the Acknowledgments appeared incorrectly due to a production error. Valentina Emiliani was also supported by the Fondation Bettencourt Schueller and AXA Research Fund, which were omitted.

The complete funding statement should read: “This work was supported by the European Union's Horizon 2020 research and innovation program under the Marie Skłodowska-Curie Grant Agreement no. 747598 (to I.-W.C.). E.P. and V.E. acknowledge support from the Agence Nationale de la Recherche ANR (Grants ANR-14-CE13-0016, Holohub and ANR-15-CE19-0001-01, 3DHoloPAc). V.E. acknowledges support from the Human Frontiers Science Program (Grant RGP0015/2016), the National Institutes of Health (Grant NIH U01NS090501-03), the Fondation Bettencourt Schueller (Prix Coups d'élan pour la recherche française), AXA Research Fund, and Getty Lab. E.R. received funding from the European Research Council Synergy Grant scheme (Helmholtz, ERC Grant Agreement 610110). The authors thank Edward S. Boyden for providing the CoChR viral constructs."

The online version has been corrected.

DOI: $10.1523 / J N E U R O S C I .1353-19.2019$ 